\title{
ArcheoSciences
}

Revue d'archéométrie

\section{The use of dilatometry in determining the manner of selecting ossuary vases: the case of Lugdunum during the Early Roman Empire}

Le recours à la dilatométrie pour déterminer le mode de sélection des vases ossuaires : le cas de Lyon durant le Haut-Empire romain

Valérie Thirion-Merle, Cécile Batigne Vallet, Christine Bonnet and Alain Bernet

\section{OpenEdition}

Journals

\section{Electronic version}

URL: http://journals.openedition.org/archeosciences/6364

DOI: 10.4000/archeosciences.6364

ISSN: 2104-3728

Publisher

Presses universitaires de Rennes

\section{Printed version}

Date of publication: 30 December 2019

Number of pages: 97-106

ISBN: 978-2-7535-8016-9

ISSN: 1960-1360

Electronic distribution by Cairn

\section{CAIRN INFO}

CHERCHER, REPÉRER, AVANCER

\section{Electronic reference}

Valérie Thirion-Merle, Cécile Batigne Vallet, Christine Bonnet and Alain Bernet, "The use of dilatometry in determining the manner of selecting ossuary vases: the case of Lugdunum during the Early Roman Empire », ArcheoSciences [Online], 43-1 | 2019, Online since 01 January 2022, connection on 02 April 2020. URL : http://journals.openedition.org/archeosciences/6364 ; DOI : https://doi.org/10.4000/ archeosciences.6364 


\title{
The Use of Dilatometry in Determining the Manner of Selecting Ossuary Vases: The Case of Lugdunum During the Early Roman Empire
}

\author{
Le recours à la dilatométrie pour déterminer le mode de sélection des vases ossuaires : \\ le cas de Lyon durant le Haut-Empire romain
}

\author{
Valérie Thirion-Merle ${ }^{a}$, Cécile Batigne Vallet ${ }^{a}$, \\ Christine Bonnet ${ }^{\mathrm{b}}$ et Alain Bernet ${ }^{\mathrm{a}}$
}

\begin{abstract}
A recent synthesis of Lyon/Lugdunum's ossuary ceramic ware showed that, typologically, the ceramics used to collect the residues of burnt bone were the same as those used in the city's kitchens (Bonnet et al., 2016). The forms are indeed strictly the same. Nevertheless, some ossuary pots from the only two funerary areas known from the Augusto-Tiberian period show particular traces totally unknown on domestic ceramics, such as slits, crumbling peeled surfaces, manufacturing flaws and other characteristics suggesting insufficient firing temperature. The authors propose the hypothesis of an opportunistic use of these pots unsuitable for culinary use. In order to check if these pots were truly underfired, we have measured about thirty ossuary ceramic ware apparent firing temperatures with a dilatometer. The elemental compositions were also determined by X-Ray fluorescence spectrometry to identify the provenance of the pots. This paper will present the results of this study and offer some interpretations of the transfer of these objects from a domestic to a funerary context.

Résumé : Une récente synthèse sur les vases ossuaires de Lugdunum a permis de montrer que typologiquement les vases utilisés pour recueillir les restes d'os brûlés étaient les mêmes que ceux utilisés dans les cuisines de la ville (Bonnet et al., 2016). Les formes sont en effet les mêmes. Mais certains vases ossuaires de deux ensembles funéraires de la période augusto-tibérienne montraient des traces inconnues en contexte domestique, telles que des fentes, des surfaces desquamées, des couleurs inhabituelles ou d'autres défauts de fabrication évoquant une température de cuisson insuffisante. Les auteurs en ont déduit une utilisation opportuniste de vases impropres à une utilisation culinaire. Pour vérifier que ces vases étaient insuffisamment cuits, des mesures de températures de cuisson par dilatométrie ont été réalisées sur une trentaine d'objets. De façon à en préciser la provenance, leur composition élémentaire a été déterminée par spectrométrie de fluorescence X. Cet article présente les résultats des mesures et propose certaines interprétations possibles pour le passage du contexte domestique au contexte funéraire de ces vases.
\end{abstract}

Keywords: dilatometry, elemental composition, Early Roman Empire, firing temperature, Lugdunum ossuary ceramic ware, X-Ray fluorescence spectrometry

Mots clés : composition élémentaire, dilatométrie, Haut Empire Romain, spectrométrie de fluorescence X, température de cuisson, vase ossuaire de Lugdunum

\footnotetext{
a Univ Lyon, CNRS, Archéologie et Archéométrie ARAR, UMR 5138, Maison de l'Orient et de la Méditerranée, 7 rue Raulin, 69007 LYON (valerie. merle@mom.fr) (mailto:cecile.batigne@mom.fr) (alain.bernet@mom.fr)

b INRAP, Archéologie et Archéométrie ARAR, UMR 5138, 12 rue Maggiorini, 69500 BRON (christine.bonnet@inrap.fr)
} 


\section{INTRODUCTION}

This study concerns a particular type of ceramic ware of the Early Roman Empire that we shall qualify as "ossuary vases". Ossuary vases are recipients intended to receive a variable quantity of burnt bone of a cremated deceased collected on a funeral pyre. These bones may have been washed before being placed in the vases. The recipient, most often ceramic, is then deposited in a permanent structure located in the same place as the pyre itself or in a pit specially dug for that purpose (Blaizot dir., 2009).

According to recent studies, in Lugdunum during the Augusto-Tiberian Period, the ossuary ceramic ware consists of exactly the same recipients as those found in domestic contexts and used in the kitchen as cooking pots intended to go on the fire. Most often, they are cooking pots of eggor globular shape with a wide but narrowed opening and covered with an adapted lid (Fig.1).

However, it was noticed that the surface finish of some of these pots used to collect burnt bone, of this precise period-that is to say from the end of the $1^{\text {st }}$ century $\mathrm{BC}$ to the beginning of the $1^{\text {st }}$ century $\mathrm{AD}$ - is very different from that of domestic ones. The pots have a damaged surface which does not correspond to that usually observed on pots in domestic or workshop contexts. Nor is this damaged appearance identified by archaeologists on pots from later periods. The peeled nature of the surface of some of the ossuary vases and other characteristics detailed below are particularly suggestive of under-firing (Bonnet et al., 2016).

The hypothesis put forward is that ceramic ware unsuitable for cooking use was selected for another use, that of collecting the burnt bones of the deceased.

To try to understand these differences and to verify if some of the pots were really underfired, we have measured apparent firing temperature with a dilatometer on ceramics found in three of Lugdunum's funerary areas. We have also tried to determine whether there were several workshops involved or a specialized one and if the ossuary ware came from already known Lugdunum workshops.

The study was made possible thanks to the extensive archaeological knowledge of the ceramic assemblages dating from the Early Roman Empire in Lyon, due to the great number of excavations of domestic and workshop contexts in the city.

\section{SAMPLE}

The sample consists of 28 sherds of ossuary vases or lids found in three of Lugdunum's funerary areas, two of the Augusto-Tiberian period and one more recent. The first site is 62 Rue du Commandant Charcot, and the second 19 Rue des Granges. These two sites are located in the $5^{\text {th }}$ arrondissement of Lyon (France) on the Fourvière hill (Fig. 2).

Fifteen globular pots and four lids came from the Rue $d u$ Commandant Charcot site (OSL 1 to OSL 19), seven globular pots and one lid from the Rue des Granges site (OSL 20 to OSL 27) (Fig.1). Some of these sherds have a damaged appearance which does not correspond to that observed on pots found in domestic contexts or in workshops (Bonnet et al., 2016). In the Rue du Commandant Charcot ensemble, the surfaces of the pots are mostly missing or they have a peeled appearance (Table 1). We observe sand grains on the surfaces and slits or cracks. Some pots seem to be underfired since they are rather soft and do not produce the sound of well fired ceramics. In the Rue des Granges ensemble, the vases are less damaged, but the surfaces seem to be worn and also look peeled. The walls or bottoms of some pots, sometimes cracked, are abnormally thin to the point that a domestic use seems excluded.

One globular pot coming from a more recent funerary site, Rues Chevrier-Rochette (OSL 28), was also included in this study. This last site, dated from the end of the $2^{\text {nd }}$ century $\mathrm{AD}$ to the $3^{\text {rd }}$ century $\mathrm{AD}$, is located in the $7^{\text {th }}$ arrondissement of Lyon. This object has a usual appearance with the same thickness, the same colour, and the same surfaces as a domestic pot. It is probably well fired. It was selected to compare a normal sherd with the damaged ones.

It is possible that the observed damages could be linked to burial conditions, but it seemed important to us to try to correlate these macroscopic observations with the technical characteristics of the vases such as the firing temperatures.

\section{EXPERIMENTAL OBSERVATIONS}

To determine their composition and try to identify their provenance, the ceramics were analysed by Wavelength Dispersive X-Ray Fluorescence Spectrometry (WD-XRF), according to the standard procedures of the Archaeology and Archaeometry Laboratory at CNRS-University of Lyon in France, using a Bruker S8 Tiger spectrometer with a Rh excitation source. This procedure gives the bulk chemical composition of the ceramic (matrix and sandy fraction) and consequently of the material used for its manufacture (Thirion-Merle, 2014; Waksman, 2014). Measurement 

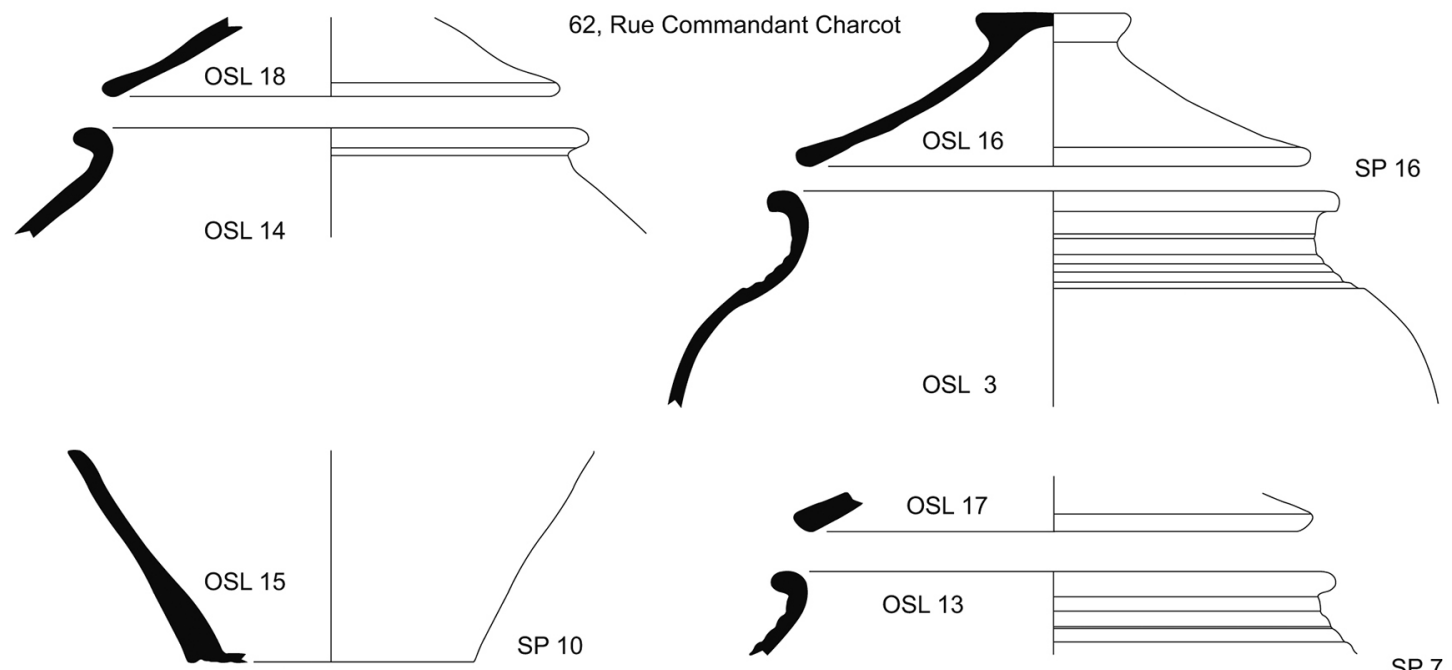

SP 7
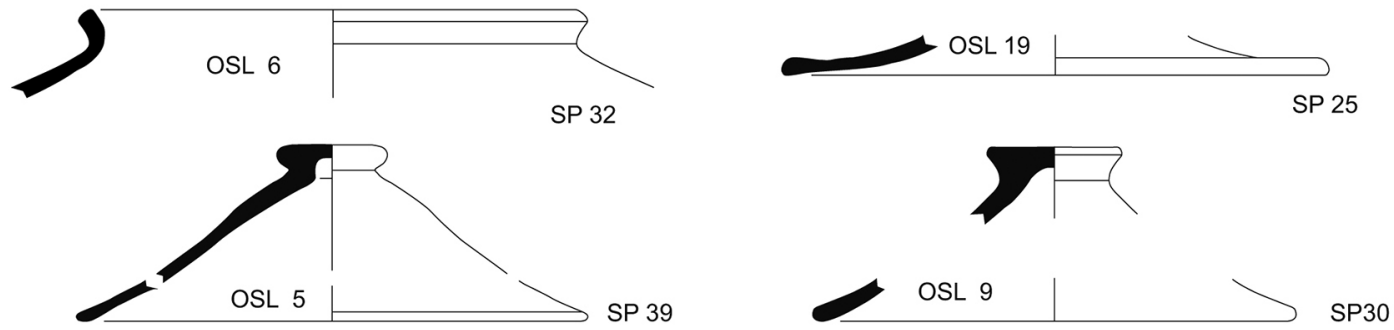

19, Rue des Granges
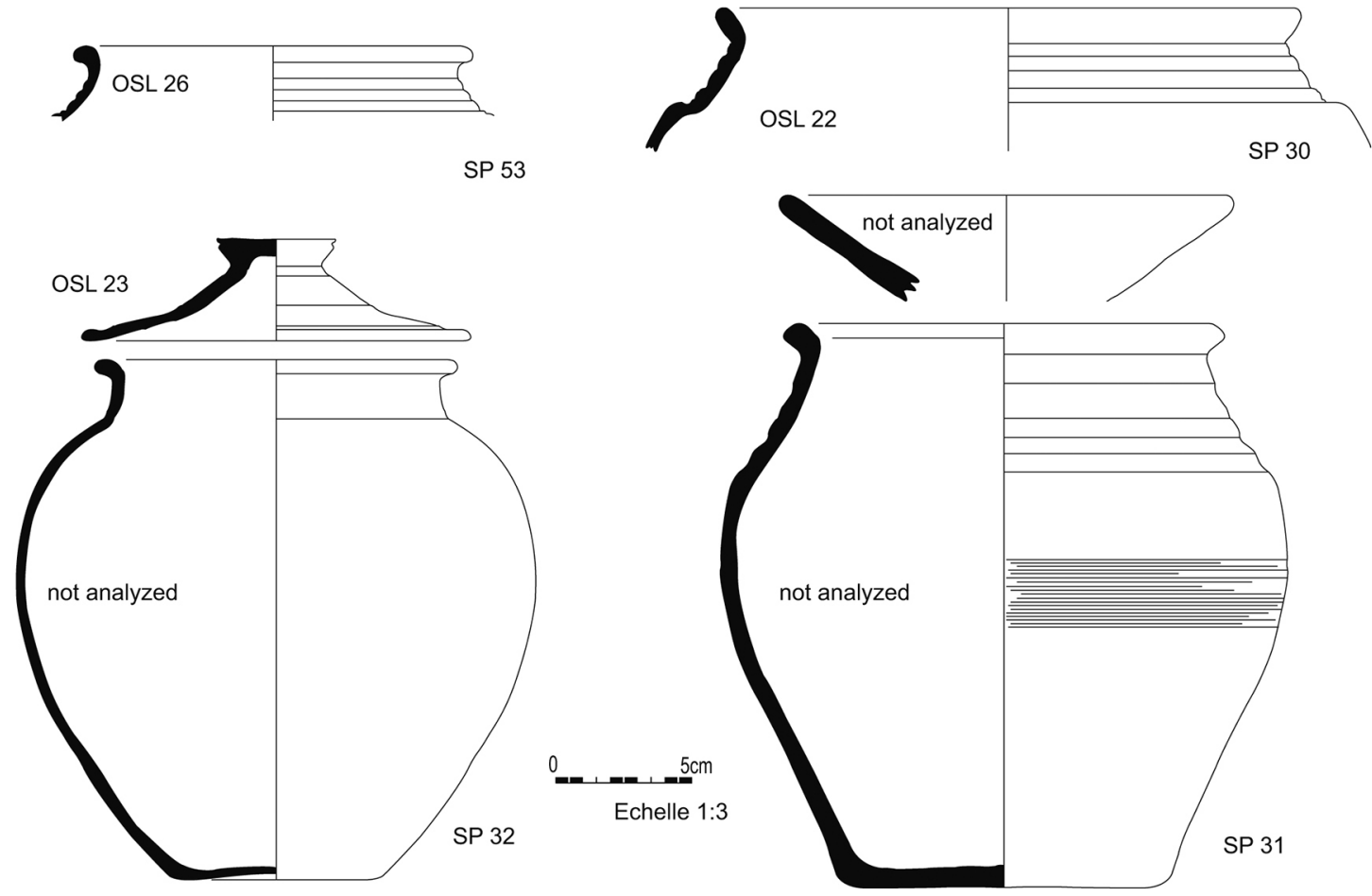

Figure 1: Selected ossuary vases from the two Augusto-Tiberian funerary ensembles (Bonnet et al., 2016). Figure 1: Vases ossuaires sélectionnés des deux ensembles funéraires Augusto-tibériens (Bonnet et al., 2016). 


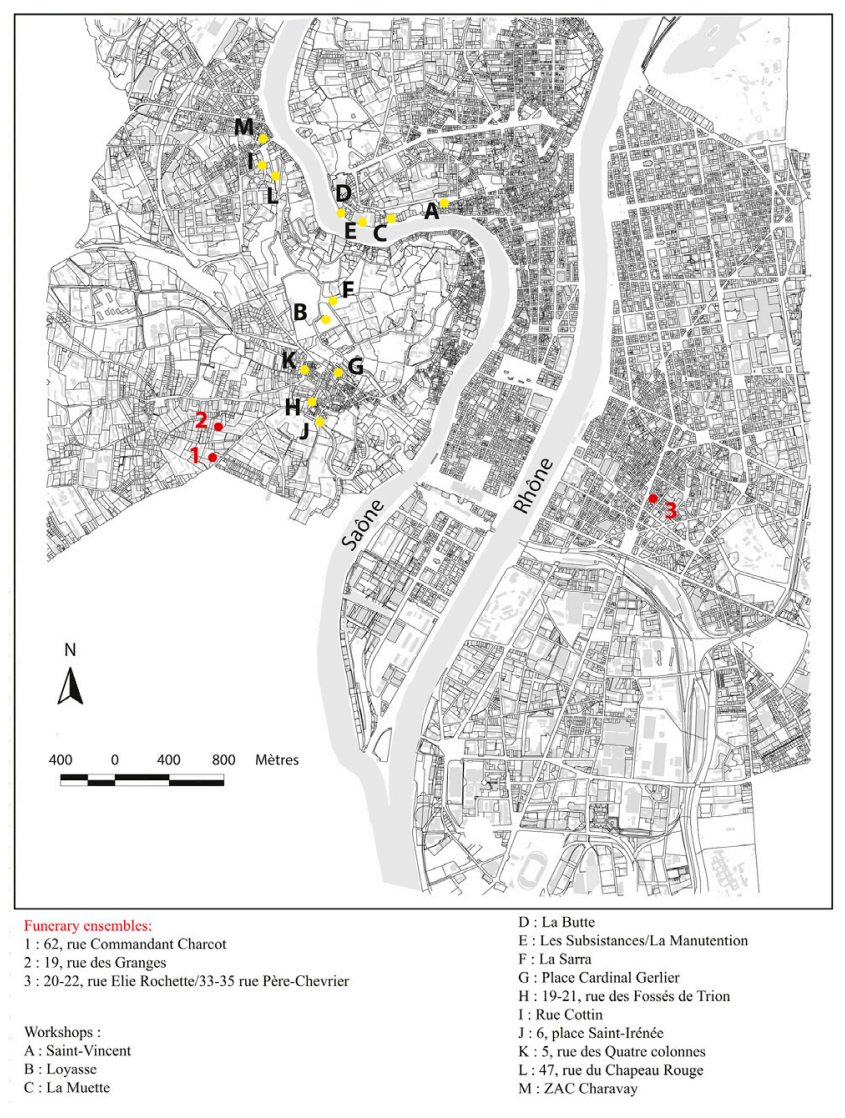

Figure 2: (See colour plate XVI) Map of the funerary ensembles selected for this study and of Lyon's ceramic workshops (Data from the French National Archaeological Map. IGN BD TOPO ${ }^{\circ}$ IGN - 2014; Direction régionale des affaires culturelles AuvergneRhône-Alpes, Service régional de l'Archéologie).

Figure 2 : (Voir planche couleur XVI) Carte des ensembles funéraires sélectionnés pour l'étude et des ateliers de potiers de Lyon (Données issues de la carte archéologique nationale. IGN BD TOPO ${ }^{\circ} I G N$ - 2014 ; Direction régionale des affaires culturelles Auvergne-RhôneAlpes, Service régional de l'Archéologie).

is carried out on glass discs of homogenous composition containing only $800 \mathrm{mg}$ of powdered sample. For each sample, twenty-four components are determined, ten majors or minors and fourteen traces. The calibration curves were established with forty international geo-standards.

The estimation of the firing temperature of the vases was done using a dilatometer measuring the thermal expansion of ceramics. This method is based on the thermal expansion properties of the ceramics and on the assumption that when clays are fired, shrinkage occurs as a result of various sintering processes (Tite, 1969). Consequently, when a fired clay ceramic is reheated from room temperature, it exhibits normal reversible thermal expansion, characteristic of its mineralogical composition. At a temperature close to the one at which the ceramic was initially fired, the physical and chemical changes occurring during its initial firing start again. Consequently, the ceramic begins to contract. The temperature at which we note this change is called "apparent firing temperature" (Fig.3a). The relationship between the apparent firing temperature and the original firing temperature depends on the mineralogical composition of the clay, the clay minerals and the impurities present in the clay as well as the time during which the highest temperature was maintained in the kiln. In general, the apparent firing temperature is not very different from that of the initial firing temperature, which is the range at which firing ended before cooling began (Cuomo di Caprio and Picon, 1999). For these measurements a Netzsch dilatometer, model 402 EP, was employed. Each sample was reheated from room temperature to $1020^{\circ} \mathrm{C}$ with a heating rate of $3 \mathrm{~K} / \mathrm{min}$. This temperature was maintained during one hour, and then the sample was cooled to room temperature at the same rate. For the acquisition of the dL-signal, a measuring range of $4000 \mu \mathrm{m}$ was selected, and consequently a resolution of $1 \mu \mathrm{m}$.

\section{ResUlts}

The compositions of the 28 sherds are given in table 1 . They reveal that all the ceramics have non calcareous fabric, and that the sample is not chemically homogeneous. Special attention was given to phosphorus oxide $\left(\mathrm{P}_{2} \mathrm{O}_{5}\right)$, a chemical component that can be trapped by ceramics in contact with bone during burial, especially in locally acidic conditions. This phenomenon depends on the nature of the ceramic (calcareous or non-calcareous) and the porosity, and could be more important for low-fired ceramics (Collomb and Maggetti, 1996; Maritan and Mazzoli, 2004; Maritan et al.,

Table 1: Summary table giving the normalized chemical compositions of Lugdunum ossuary pots, obtained by WD-XRF, in the same order as the Figure 4 dendrogram (majors and minors are given in percentage of oxide, trace elements in parts per million of metal), the apparent firing temperatures and the damage observed on the wares $(\mathrm{RNP}=$ Ribbed neck pot; $\mathrm{ASP}=$ Angular shouldered pot; $\mathrm{L}=$ rounded lip lid).

Tableau 1 : Tableau récapitulatif donnant les compositions chimiques normalisées des vases ossuaires obtenus par WD-XRF dans l'ordre du dendrogramme de la figure 4 (Les concentrations des majeurs et mineurs sont exprimées en pourcentage d'oxyde, les éléments traces en parties par million), les températures apparentes de cuisson et les dommages observés sur les vases $(R N P=$ pot à col côtelé; $A S P=$ pot à épaule carénée; $L=$ couvercle à lèvre arrondie). 


\begin{tabular}{|c|c|c|c|c|c|c|c|c|c|c|c|c|c|c|c|c|c|c|c|c|c|c|c|c|c|c|c|c|}
\hline 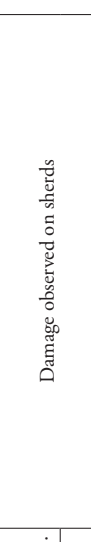 & 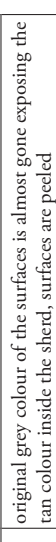 & 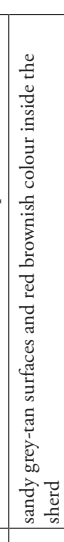 & 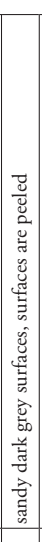 & & 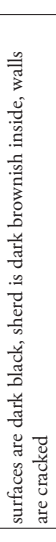 & 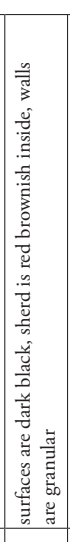 & 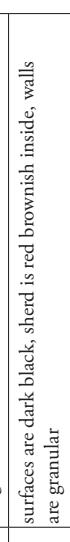 & 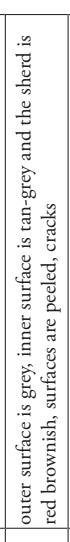 & 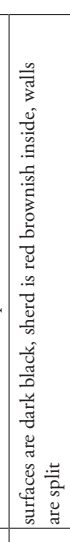 & 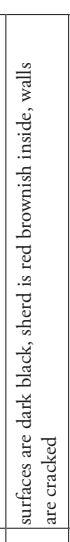 & 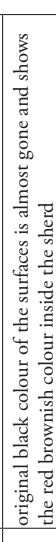 & 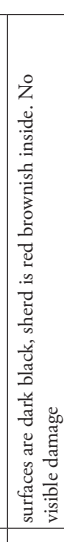 & 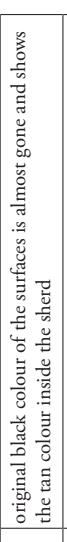 & 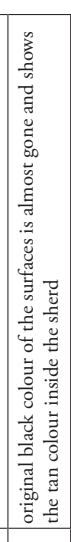 & 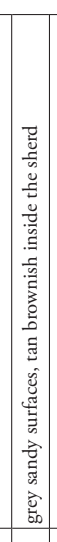 & 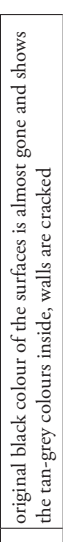 & 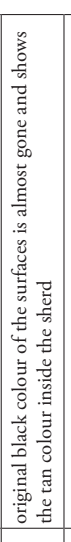 & 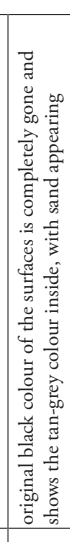 & 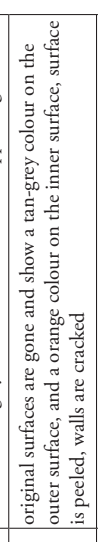 & 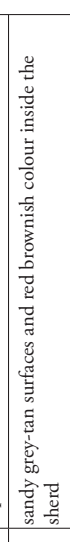 & 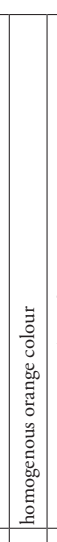 & & & 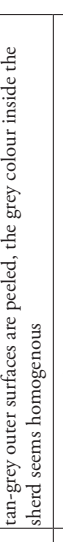 & . & 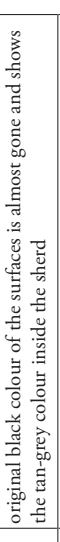 & 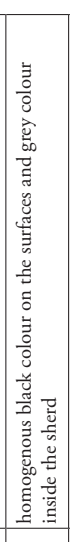 & \\
\hline $\begin{array}{l}0 \\
0 \\
0 \\
0 \\
0\end{array}$ & ळે & $\underset{\infty}{\hat{\infty}}$ & 文 & $\mid \begin{array}{c}9 \\
\infty \\
\infty\end{array}$ & 䙳 & 范 & 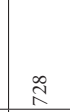 & 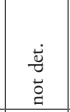 & त्तु & శ్రి & 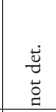 & 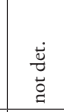 & త్రి & ఫ్రి & 总 & 范 & శ్ర & त్రి & $\stackrel{8}{\infty}$ & $\$$ & $\mid \begin{array}{c}+ \\
\infty\end{array}$ & \begin{tabular}{|l|}
$\infty$ \\
\multirow{2}{*}{}
\end{tabular} & ह & $\check{\Sigma}$ & & 菅 & $\underset{\infty}{\infty}$ & \\
\hline J & & $\hat{m}$ & 東 & 茾 & 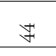 & - & $\hat{x}$ & & 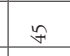 & & 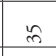 & $\mathrm{N}$ & $\bar{z}$ & 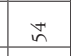 & 81 & F & & $\nexists$ & & $\infty$ & $\ddot{\Omega}$ & 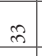 & & 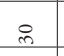 & $\approx$ & & a & \\
\hline$\stackrel{2}{\sharp}$ & f & ta & \& & $\vec{F}$ & 6 & & in & & $\approx$ & & $\approx$ & & & & 41 & & & & & & o & $\infty$ & i & 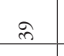 & & & & \\
\hline$F$ & & $\infty$ & 3 & $\stackrel{\infty}{\simeq}$ & & & $\stackrel{\infty}{\sim}$ & & 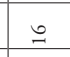 & & 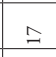 & & & & 81 & & & & & 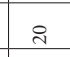 & $\dot{\sim}$ & $\stackrel{\infty}{ }$ & 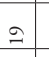 & 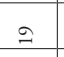 & 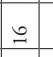 & & & \\
\hline$>$ & & 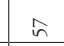 & $\hat{n}$ & $a$ & $N$ & & f & & $\grave{\imath}$ & & $a$ & & & & $\approx$ & $\approx$ & & & & $\sigma$ & \& & $\infty$ & 5 & बે & ○ & & & \\
\hline 8 & $\exists$ & 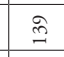 & פุ & $\checkmark$ & & & 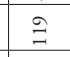 & & $\approx$ & & $F$ & & & & $\triangleq$ & 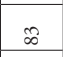 & & & & & $\exists$ & $\exists$ & & & & & & \\
\hline$>$ & $\sigma$ & $\stackrel{2}{\varrho}$ & 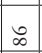 & I & $\stackrel{m}{\exists}$ & $\propto$ & E & $n$ & tot & & $=$ & & $=$ & & 2 & 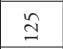 & & & & & 4 & \& & \& & 。 & ڤ & & $\stackrel{m}{2}$ & \\
\hline 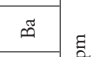 & 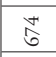 & : & శ్రి & 6 & $\overrightarrow{\underline{0}}$ & $\stackrel{\infty}{\wedge}$ & $\stackrel{\infty}{\stackrel{\infty}{\Perp}}$ & हे & in & & f) & $\forall$ & $\infty$ & ల్ల & 命 & $\tilde{\sigma}$ & & & & F & \begin{tabular}{|l|}
\multirow{\alpha}{*}{} \\
\end{tabular} & I & : & $\underset{\sim}{\infty}$ & $\sqrt{2}$ & 亲 & & \\
\hline 3 & $n$ & 8 & 6 & 8 & $\pi$ & $i$ & $\stackrel{\infty}{0}$ & $\hat{m}$ & $f$ & & ते & & $\approx$ & $\stackrel{n}{n}$ & $\forall$ & F & $n$ & 5 & & $\infty$ & $\approx$ & $\therefore$ & $n$ & $q$ & H & & ir & \\
\hline$\ddot{z}$ & \& & st & in & $\vec{n}$ & $i$ & 8 & $\vec{\infty}$ & & $\vec{n}$ & & in & & $y$ & & $\mathscr{F}$ & & & & & - & of & $\infty$ & $i$ & f & $\approx$ & & & \\
\hline ப & $\Xi$ & 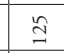 & 4 & 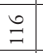 & 8 & & $\vdots$ & & $\stackrel{v}{g}$ & & $\exists$ & & $=$ & & -1 & & & & & & 8 & 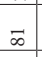 & ه & d & A & & & \\
\hline 歨 & 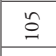 & 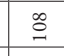 & $=$ & $\mathscr{d}$ & & S & st & & r & & $\infty$ & & 8 & $\infty$ & al & $\circ$ & & & & $\Xi_{0}^{+}$ & 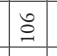 & $\hat{m}$ & 아 & in & $\widetilde{\delta}$ & & & \\
\hline$\vec{\approx}$ & . & 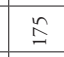 & 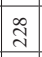 & $\stackrel{\infty}{\stackrel{\infty}{\infty}}$ & 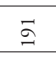 & $\Phi$ & $\square$ & & & & $\infty$ & & $\alpha$ & & in & 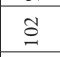 & & & & & in & ธ & ชู & $n$ & 涪 & & $\infty$ & \\
\hline$\dot{s}$ & $\stackrel{\infty}{\leftrightarrows}$ & $\stackrel{\bullet}{\leftrightarrows}$ & $\stackrel{6}{\because}$ & $\cong$ & $\stackrel{8}{0}$ & & : & & $A$ & & & & & & 10 & & & & & 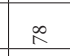 & $a_{-}$ & $i$ & 5 & - & $a$ & & & \\
\hline N & $\stackrel{\circ}{m}$ & 商 & $\overrightarrow{\tilde{m}}$ & సิ & ¿े & $\ddot{m}$ & 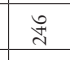 & $\approx$ & $\bar{m}$ & & Fे & & $\infty$ & $m$ & D. & $\partial$ & & $\approx$ & & ले & 焉 & if & i & in & 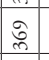 & & $\hat{\mathrm{y}}$ & \\
\hline $0^{n}$ & 0 & 5 & \begin{tabular}{|l}
8 \\
fo \\
0
\end{tabular} & 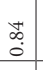 & రి & $\stackrel{\infty}{\curvearrowleft}$ & $n$ & 8 & & & in & & 0 & ని & $\widehat{人}$ & 0 & & $\hat{n}$ & & $\begin{array}{l}\overrightarrow{5} \\
0\end{array}$ & $\mid \begin{array}{c}\infty \\
0 \\
0\end{array}$ & 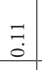 & 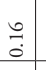 & 0 & 0 & & a & \\
\hline $\begin{array}{l}0 \\
z^{-10}\end{array}$ & $=$ & 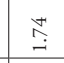 & 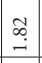 & $\stackrel{\infty}{\rightrightarrows}$ & $\mathfrak{x}^{\infty}$ & $\vec{i}$ & & $\tilde{3}$ & & & 0 & & & & & 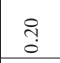 & & & & & $\begin{array}{l}\infty \\
0 \\
0\end{array}$ & $\stackrel{3}{3}$ & নु & aे & - & & $\dot{0}$ & \\
\hline $\begin{array}{l}\circ \\
\frac{\xi}{2}\end{array}$ & 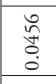 & $\begin{array}{l}\text { 略 } \\
\text { o. }\end{array}$ & 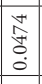 & \begin{tabular}{|c|c|}
$\vec{n}$ \\
$\vdots$ \\
0 \\
0
\end{tabular} & $\tilde{s}^{\circ}$ & a & 兽 & 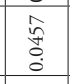 & $\begin{array}{l} \\
\text { o } \\
0 \\
0 \\
0\end{array}$ & & $\begin{array}{l}\stackrel{0}{0} \\
0 \\
0 \\
\end{array}$ & & 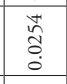 & $\frac{0}{2}$ & \begin{tabular}{|l|} 
\\
0 \\
0 \\
0 \\
0
\end{tabular} & $=$ & $\begin{array}{l}\text { no } \\
\vdots \\
0 \\
0\end{array}$ & & $\check{\beth}$ & $\begin{array}{l}\text { مे } \\
\text { ô. }\end{array}$ & $\begin{array}{l}\infty \\
\infty \\
0 \\
0 \\
\end{array}$ & 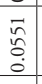 & n & $\begin{array}{l} \\
\vdots \\
0 \\
0\end{array}$ & $\mid \begin{array}{l}0 \\
0 \\
0 \\
0 \\
0\end{array}$ & 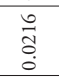 & $\begin{array}{l}\overrightarrow{\vec{n}} \\
\stackrel{0}{0} \\
0\end{array}$ & \\
\hline $\begin{array}{l}\circ \\
\\
\end{array}$ & के & $\stackrel{f}{\rightarrow}$ & 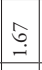 & $\stackrel{\infty}{-}$ & $\stackrel{8}{\leftrightarrow}$ & $\stackrel{\mathscr{ٌ}}{\rightarrow}$ & - & 号 & & & $\tilde{z}$ & & 0 & $\tilde{\tilde{O}}$ & & & & & & & 0 & 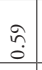 & 0 & 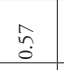 & 0 & & 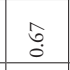 & \\
\hline $\begin{array}{l}0^{\prime \prime} \\
\mathbb{C}^{\prime \prime}\end{array}$ & $\frac{7}{7}$ & $\underset{\sim}{+}$ & $\stackrel{\substack{\infty \\
\vec{\sim}}}{ }$ & 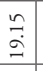 & $\begin{array}{l}0 \\
\stackrel{3}{N}\end{array}$ & $\begin{array}{l}\hat{n} \\
\stackrel{\Omega}{\Omega}\end{array}$ & ¿̊. & D & & & $\infty$ & & & & 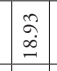 & 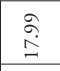 & & 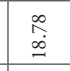 & & 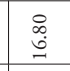 & $\begin{array}{c}\tilde{\hat{n}} \\
\grave{n}\end{array}$ & \begin{tabular}{|c|c|} 
\\
0 \\
\\
\end{tabular} & 苞 & $\begin{array}{l}\stackrel{b}{0} \\
\end{array}$ & $\begin{array}{l}\tilde{v} \\
\tilde{n} \\
\end{array}$ & & 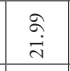 & \\
\hline $\mathrm{O}^{\prime \prime}$ & $\sqrt{6}$ & $\begin{array}{l}2 \\
\stackrel{1}{6} \\
\end{array}$ & \begin{tabular}{|c|} 
\\
0 \\
0 \\
0
\end{tabular} & 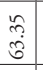 & בֶ. & 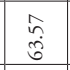 & & $\sqrt{2}$ & & & & & & ه্ & & 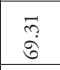 & & $\%$ & & 8 & \begin{tabular}{|c|}
7 \\
8
\end{tabular} & 10 & a & $\begin{array}{ll}z_{1}^{*} \\
\end{array}$ & 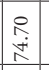 & 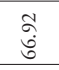 & 8 & \\
\hline 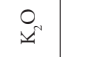 & $m$ & 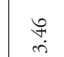 & 芦 & $\stackrel{d}{a}$ & స్ & $m$ & $\tilde{}$ & $\stackrel{n}{\rightarrow}$ & I & & $\therefore$ & & $n$ & $\forall$ & $n$ & gे & & 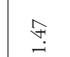 & & $\stackrel{\Delta}{\stackrel{H}{*}}$ & $\begin{array}{l}\check{a} \\
o\end{array}$ & $0^{\circ}$ & 0 & - & $\stackrel{\infty}{=}$ & & - & \\
\hline 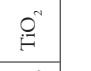 & $a$ & ఫ్తి & 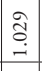 & త্ণ & 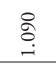 & 0 & & 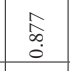 & & & $\begin{array}{c}\substack{\infty \\
\infty \\
\infty \\
0 \\
0} \\
\end{array}$ & & $0_{0}^{\infty}$ & 今े & $\vec{n}$ & $\begin{array}{c}+\infty \\
\infty \\
0 \\
0\end{array}$ & & & & $\begin{array}{l}\hat{h} \\
\hat{c} \\
0\end{array}$ & $\mid \begin{array}{c}\pi \\
2 \\
0\end{array}$ & 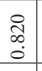 & 0 & 尾 & \begin{tabular}{|c|c}
$\infty$ \\
$\infty$ \\
0 \\
0
\end{tabular} & & $\vec{D}$ & \\
\hline फ & 0 & 0 & $\therefore$ & $\stackrel{9}{\infty}$ & $\stackrel{\circ}{\circ}$ & 6 & 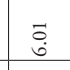 & तु & & & 6 & & 6 & 6 & & $1:$ & $r$ & & & $\overrightarrow{\tilde{n}}$ & & & nn & is & $\overrightarrow{6}$ & $\infty$ & $\infty$ & \\
\hline ఊ & 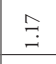 & $\stackrel{I}{\exists}$ & $\stackrel{ \pm}{ت}$ & $\stackrel{\infty}{\exists}$ & $\stackrel{\mathscr{n}}{\rightarrow}$ & - & & $\infty$ & & & తి & & $\hat{\curvearrowright}$ & $\approx$ & & $0^{\circ}$ & & & & 0 & & & 8 & 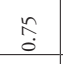 & $\tilde{o}$ & న్ & $\stackrel{\infty}{\infty}$ & \\
\hline & $\underline{g}$ & 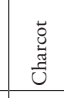 & 4 & 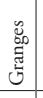 & 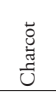 & 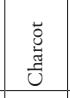 & 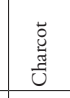 & 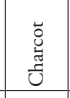 & & & 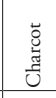 & & & 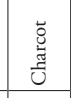 & & 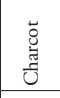 & & & & 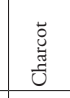 & $t$ & & & 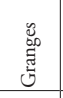 & 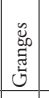 & 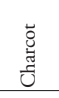 & 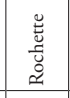 & \\
\hline & $\hat{\bar{n}}$ & & & & $\bar{\partial}$ & 单 & & $<$ & & & & & & & «1 & & & & & ¿ & & & & & & & & \\
\hline & $\infty$ & $\overrightarrow{\vec{b}}$ & & & $\vec{y}$ & 8 & 0 & & & & $\stackrel{\infty}{=}$ & & & $\begin{array}{l}\overrightarrow{7} \\
\overrightarrow{3}\end{array}$ & & $\begin{array}{l}\vec{t} \\
\overrightarrow{0}\end{array}$ & & & & $\exists$ & $\vec{\jmath}$ & & & & & & t. & \\
\hline
\end{tabular}




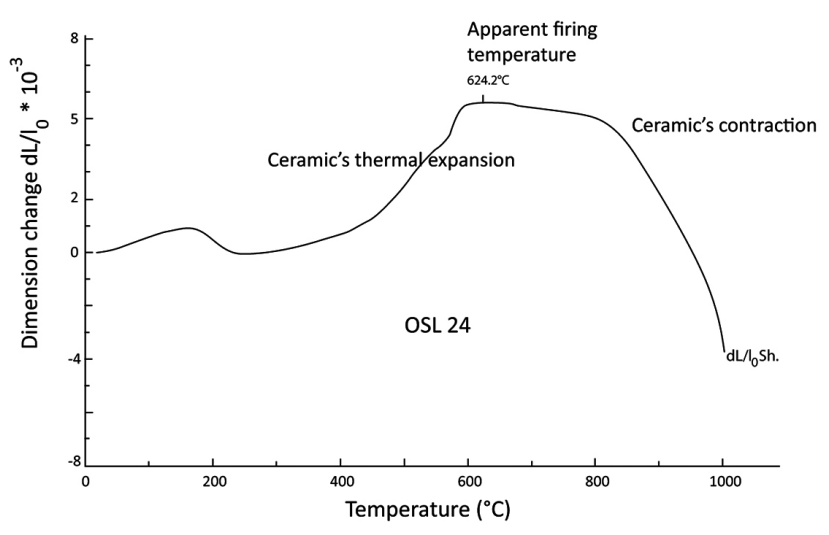

(a)

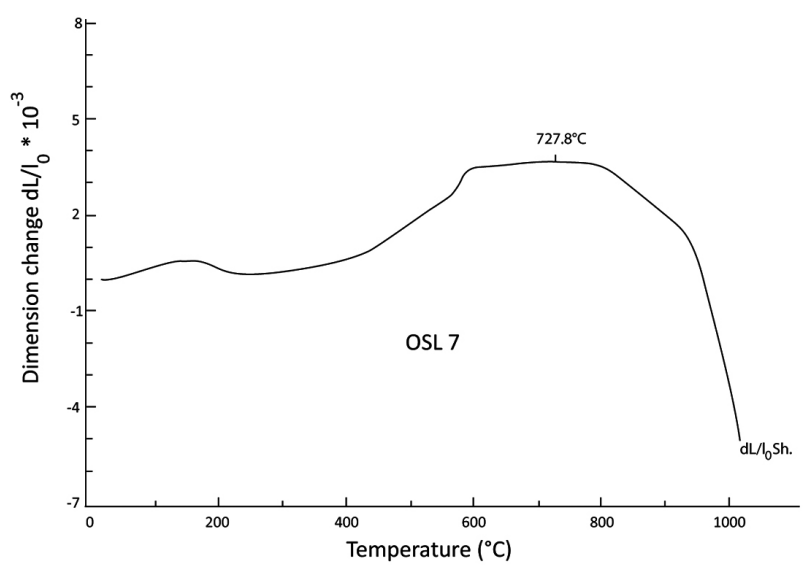

(c)

Figure 3: Thermal expansion curves recorded for four ossuary pots. Figure 3: Courbes de dilatation enregistrées pour 4 vases ossuaires.

2009). It is generally correlated with higher barium content and the leaching of other elements (Schneider, 2017). This could consequently affect the bulk ceramic composition and induce problems in determining the origin. However, in some cases, the occurrence of phosphates in the samples does not significantly influence their bulk chemical composition (Maritan and Mazzoli, 2004). In our case, the $\mathrm{P}_{2} \mathrm{O}_{5}$ contents vary between 0.2 and 0.8 wt. \%. Only one sherd has a content of about $1 \mathrm{wt} \%$ (OSL 3) and may therefore have been altered. For the others, phosphorus contamination is not obvious, especially since barium levels are not particularly high. These values are lower than might have been expected from ceramics in contact with bones, even if a threshold for contamination is often difficult to fix. In clay, the phosphorus content varies generally from $0.2 \%$ to $0.5 \mathrm{wt} . \%$, whereas in the ceramics produced from it, the content is slightly higher due to the simple loss on ignition from the firing (Collomb and Maggetti, 1996). Only a few

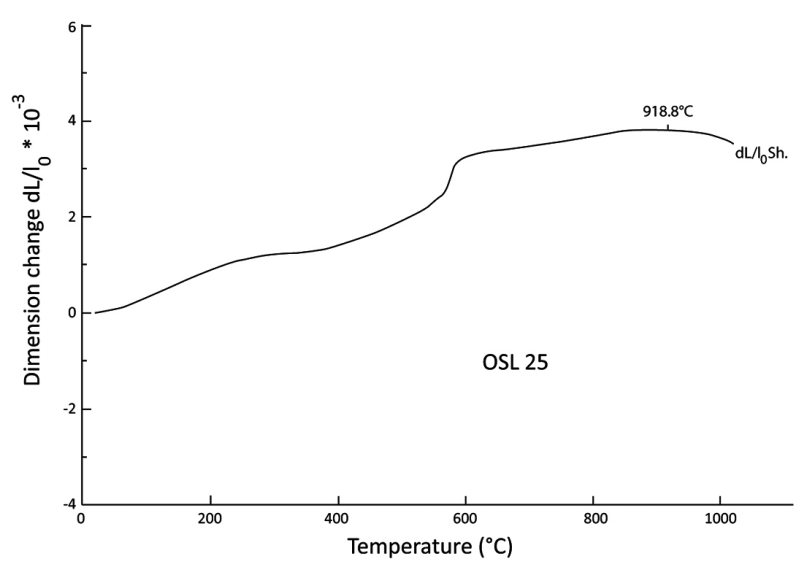

(b)

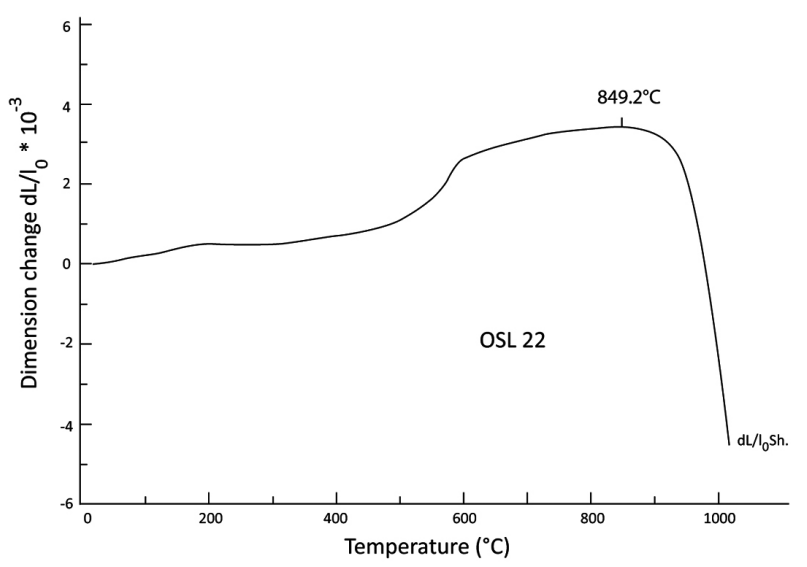

(d)

vases could have been affected by phosphorus contamination. Nevertheless, attention will be paid to this point later when using the data.

In order to classify the analyses and to test resemblances, multivariate statistical data treatment was processed. The method used is a hierarchical clustering analysis, applied to reduced centred data, using Euclidian distance and average linkage (Picon, 1984; Baxter, 1994). In our case, the calculations were carried out on 17 of the 24 determined chemical components: 8 major and minor elements in ceramics $\left(\mathrm{MgO}, \mathrm{Al}_{2} \mathrm{O}_{3}, \mathrm{SiO}_{2}, \mathrm{~K} 2 \mathrm{O}, \mathrm{CaO}, \mathrm{TiO}_{2}, \mathrm{MnO}, \mathrm{Fe}_{2} \mathrm{O}_{3}\right.$ ) and 9 trace elements of various geochemical behaviour $(\mathrm{V}, \mathrm{Cr}$, $\mathrm{Ni}, \mathrm{Zn}, \mathrm{Rb}, \mathrm{Sr}, \mathrm{Zr}, \mathrm{Ba}$, and $\mathrm{Ce})$. Phosphorus oxide $\left(\mathrm{P}_{2} \mathrm{O}_{5}\right)$, lead $(\mathrm{Pb})$ and copper $(\mathrm{Cu})$ were not considered for classification since they are indicative of possible pollution problems; sodium oxide $\left(\mathrm{Na}_{2} \mathrm{O}\right)$, lanthanum $(\mathrm{La})$, yttrium $(\mathrm{Y})$, thorium (Th) lack relevance for the comparisons. The cluster 
analysis of these 28 sherds shows at least four compositional groups and many isolated samples (Fig. 4).

The first two groups (named A1 and A2), as well as the two isolated samples situated between them, present similar chemical characteristics, and can be distinguished from the $\mathrm{B}$ groups by higher contents of alumina, potassium oxide, magnesium oxide and sodium oxide as well as higher contents of strontium, rubidium, chromium and cerium (Table 1).

The two other groups (B1 and B2) comprising 18 samples, as well as the isolated sample between them, have also similar chemical characteristics, particularly high content of silica and lower content of titanium oxide (Table 1). With

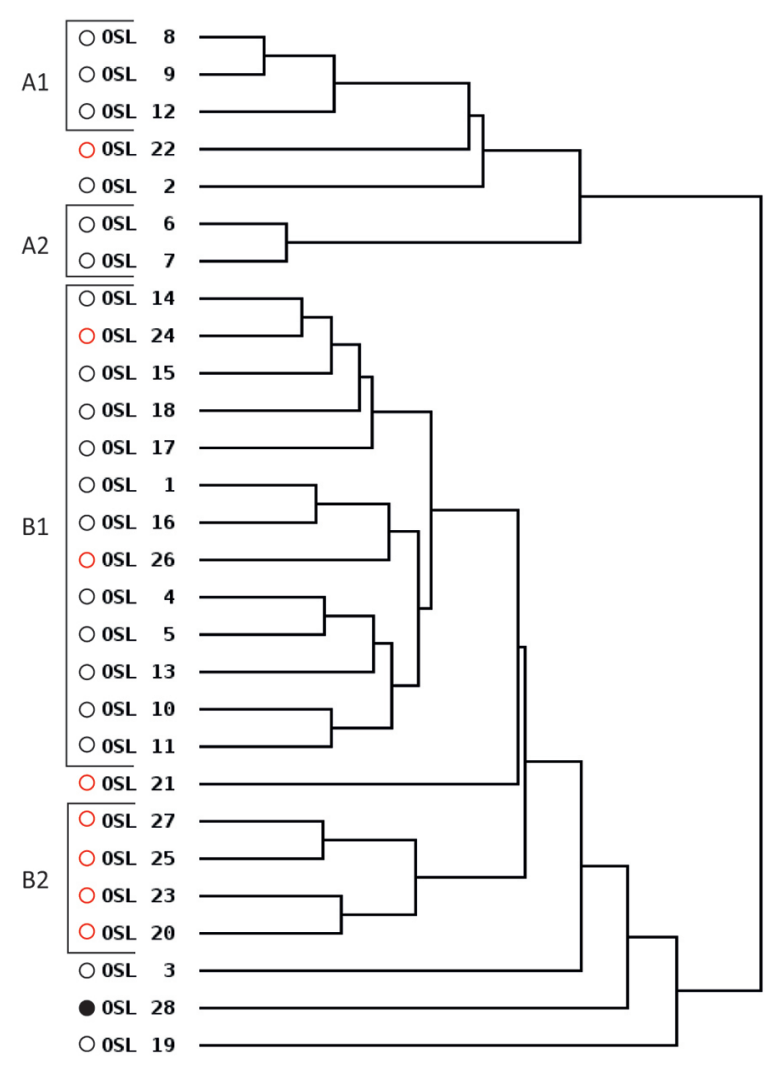

\begin{tabular}{|l}
\hline 62, Rue du Commandant Charcot \\
19, Rue des Granges \\
Rues Chevrier-Rochette
\end{tabular}

Figure 4: (See colour plate XVI) Dendrogram of cluster analysis of the 28 ossuary vases studied - calculations based on 17 chemical components.

Figure 4: (Voir planche couleur XVI) Classification ascendante hiérarchique des 28 vases ossuaires étudiés; calculs réalisés sur 17 composants chimiques. the exception of group B2, exclusively composed of samples found in the Rue des Granges site, the groups are composed of samples found at both sites. The marginal position of the OSL 3 sample could possibly be explained by an altered composition linked to the burial conditions insofar as the contents of elements such as phosphorus, zinc, copper and also chromium are relatively high.

The more recent sample discovered at the Rues ChevrierRochette site (OSL 28) is isolated at the end of the dendrogram and presents a completely different composition, suggesting another clay material and another provenance.

Consequently, there are more than four different clay materials used to produce these ossuary pots.

A final remark concerning the composition of these vases: for three of them, we note abnormally high content in copper (more than $130 \mathrm{ppm}$ ) which is higher than the natural content in clay. It might be due to the presence in the pots of metallic parts which could have contaminated the ceramic. However, no information about metallic objects was mentioned in the excavation reports.

The apparent firing temperature was determined for only 23 of the 28 samples (Table 1) due to practical reasons: some sherds were too small to be sampled for the dilatometer measurement. Thus, the apparent firing temperature of three sherds of the Rue du Commandant Charcot site (OSL 14, OSL 17 and OSL 18) and two of the Rue des Granges site (OSL 20, OSL 26) was not determined. We recorded the thermal expansion curves of the different samples and determined their apparent firing temperatures. We observed different profiles of the thermal expansion curves, suggesting that the sintering processes do not occur at the same temperature range. We got a curve profile for samples having low apparent firing temperature (below $650^{\circ} \mathrm{C}$, Fig.3a). Samples whose apparent firing temperatures are above $900^{\circ} \mathrm{C}$ present a totally different curve profile (Fig.3b). Samples with intermediate apparent firing temperatures present two similar curve profiles (Fig.3c and 3d).

We also made a histogram of the apparent firing temperatures of these ceramic samples from a funerary context (Fig5a) in which we indicate the chemical group to which each sample belongs. These apparent temperatures are spread between $620^{\circ} \mathrm{C}$ and $950^{\circ} \mathrm{C}$. They cover a wide range and are highly variable. Some samples are not very well fired and indeed markedly less fired than others. In particular, we notice that most samples of chemical group B1 are much underfired, while those of group B2 are highly fired. As for the samples belonging to groups $\mathrm{A}$, they present a large variety of firing temperatures, from $640^{\circ} \mathrm{C}$ to $880^{\circ} \mathrm{C}$. We can conclude that, for these two funerary contexts, the apparent firing temperatures are not homogeneous, varying from low to high. 
In a previous study, apparent firing temperatures were measured on cooking wares found in domestic contexts in Lugdunum, also situated in the $5^{\text {th }}$ arrondissement and dating between La Tène finale and the $3^{\text {rd }}$ century AD. The selected samples were all produced in local workshops (Batigne Vallet, 1999). The author favoured measurements on samples from consumption contexts rather than workshop contexts, because wares found in workshops might have been rejected by the potters due to firing problems. Remarkably, in domestic ensembles, very few samples present apparent firing temperatures below $700^{\circ} \mathrm{C}$. We can deduce that traditionally in Lugdunum at this period the pots used for cooking were fired at temperatures ranging from 750 and $950^{\circ} \mathrm{C}$ (Fig 5b).

\section{Discussion}

From the chemical compositions, we can deduce that the ossuary pots of our sample were made with many dif- ferent clay materials suggesting various workshops and consequently various supplies.

We then tried to determine the origin of these vases and to compare them, first of all, with those of local workshops.

In Lugdunum, 13 ceramic workshops dating from the middle of the $1^{\text {st }}$ century B.C. to the $4^{\text {th }}$ century A.D., are known (Desbat, 2010). They are distributed in three areas: on the right bank of the Saône in the Vaise district, on the left bank of the Saône and on Fourvière hill (Fig.2). Numerous studies have been carried out on these workshops and their productions, and several reference groups created based on chemical composition to characterize the different productions (Desbat et al., 1996; Desbat et al., 1997, Batigne Vallet dir.).

Our ossuary samples were compared to the references established for five of these workshops: those which produced non-calcareous common ware and in particular the type of cooking pots corresponding to the ossuary vases which have been chemically characterized (workshops F, G, K, L and M of fig.2). Chronologically, the workshops named La Sarra

\section{a) Funerary contexts}

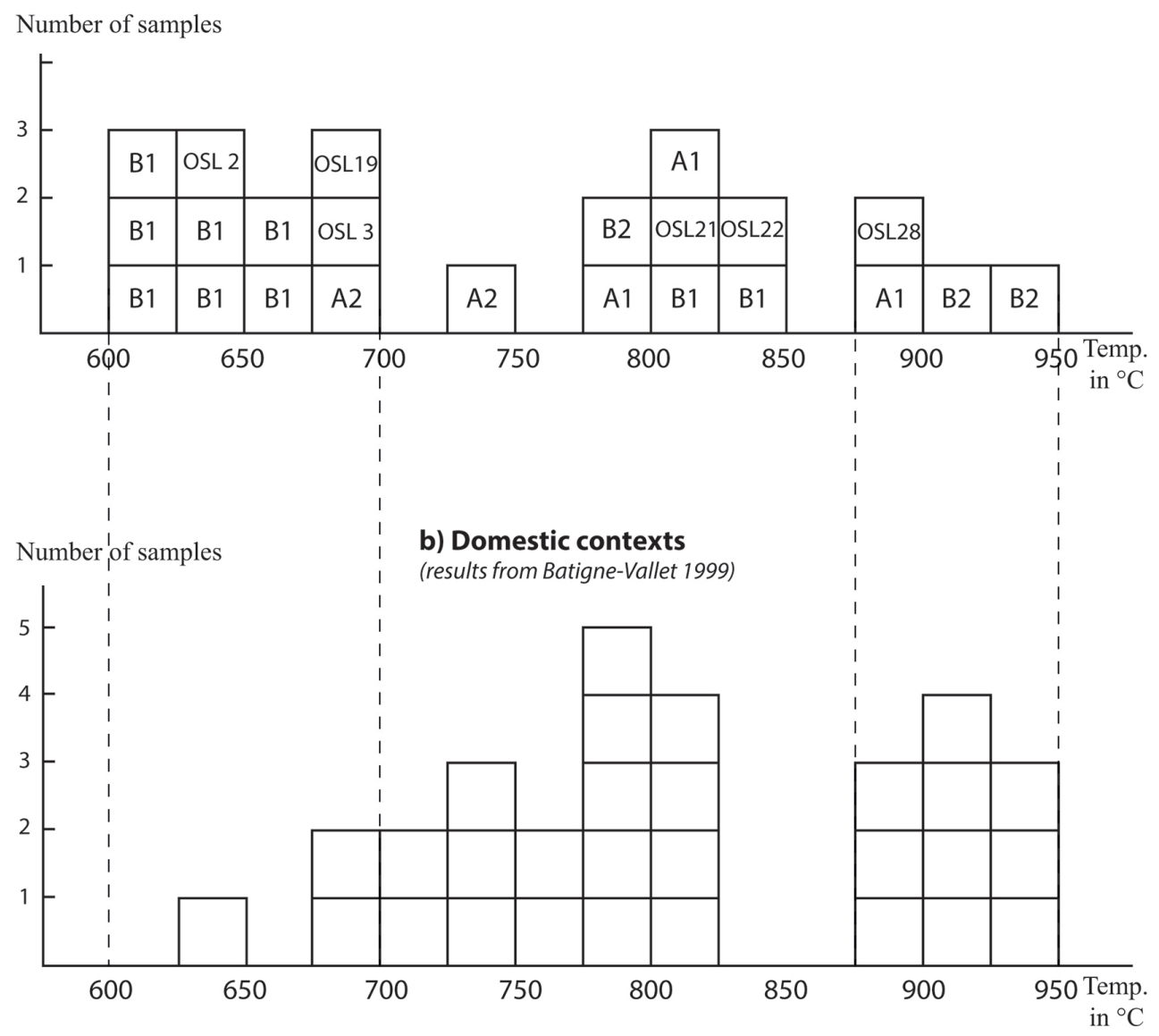

Figure 5: Histograms of apparent firing temperatures (For each sample either the compositional group to which it belongs or its analysis number is given).

Figure 5: Histogrammes des températures de cuisson apparentes (Pour chaque échantillon est indiqué soit le groupe de composition auquel il appartient soit son numéro d'analyse). 
(F) and Cardinal-Gerlier (G) correspond to the two funerary areas studied.

The dendrogram of the new classification is given in figure 6. The A groups' samples form a separate group at the end of the dendrogram, suggesting completely different compositions. Examination of the compositional data of the Lugdunum workshops' references and those of the A groups' samples shows that the latter were not produced by one of the 5 selected workshops. Comparisons made with all analysed common ware coming from domestic contexts in Lugdunum (Batigne Vallet, 1999) and other regional workshops (Batigne Vallet dir.) show that, in terms of chemical composition, there exists a group of common ware including our ossuary vases of the A groups that could have been produced by a yet-to-be-identified workshop located in Lyon or to the south, along the Rhône near Saint-Romain-en-Gal.

The ceramics belonging to group B2 fit into the Lyon references. Their chemical compositions are very similar to some Lyon references, especially those corresponding to the workshop "La Sarra" which is, as already mentioned, contemporary.

The ossuary vases of group B1 form a distinct group among the Lyon references. Their compositions show differences in elements such as chromium or zirconium. We can ask if their compositions might have been subject to variations due to their burial conditions, or if they represent a very limited production run of one of the Lugdunum workshops. They might also correspond to an as-yet-unidentified and analysed Lugdunum workshop. Indeed, as for the vases of the A groups, they correspond to the compositions of another group of common ware from Lugdunum's domestic contexts which have also been attributed to a Lyon production on archaeological grounds (Batigne Vallet, 1999). Moreover, they are totally different from the compositions of all the identified and analysed regional workshops, as are the vases of group B2 (Batigne Vallet dir.). Consequently, the most likely hypothesis is that all these different ossuary vases from groups B1 and B2 were produced in Lugdunum workshops.

Thus, the ossuary vases used in Lugdunum during the Augusto-Tiberian period do not come from a single specialised workshop. The supply is varied, with the pots coming from different local workshops as well as from more distant ones.

From the study of the apparent firing temperatures, we noted that the ossuary vases of group B2 are fired at a higher temperature than the others. They were probably diverted from their first function, namely the cooking of food, for a reason other than poor quality due to bad firing. On the other hand, most of the B1 samples are underfired and pre-
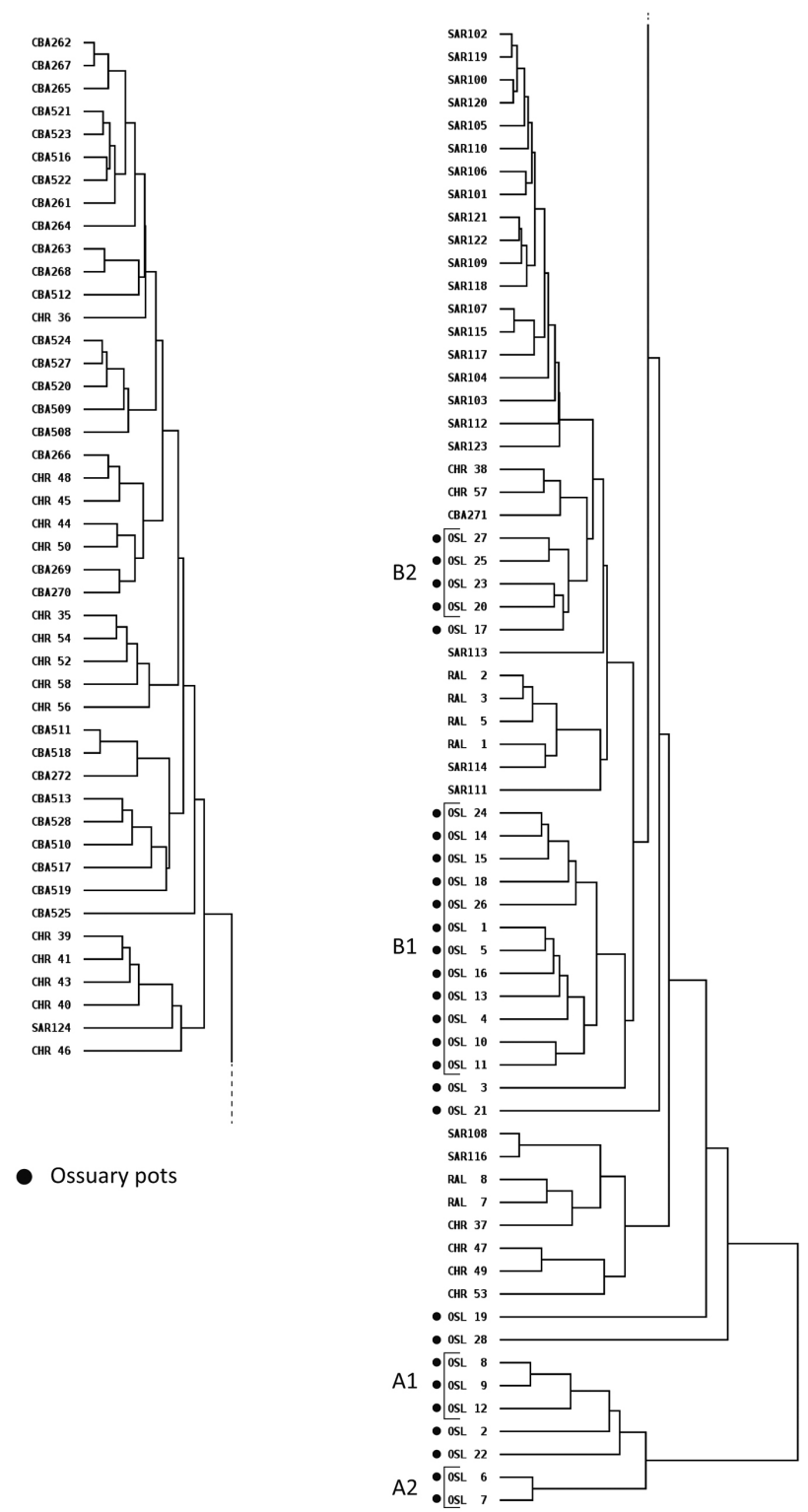

Figure 6: Dendrogram of cluster analysis of the 28 ossuary vases studied and the Lugdunum references, calculated on 14 chemical components ( $\mathrm{Zn}, \mathrm{Ba}$ and Ce have not been used due to pollution problems with the reference samples).

Figure 6: Classification ascendante hiérarchique des 28 vases ossuaires et des références des ateliers lyonnais; calculs réalisés sur 14 composants chimiques ( $\mathrm{Zn}, \mathrm{Ba}$ et Ce nont pas été retenus du fait de problèmes de pollution des échantillons références).

sent very bad surface finishes, and are hence unsuitable for cooking. However, the potters did not want to lose their production and therefore sold it for some other purpose, such as funerary use, as a second choice. Concerning the vases of groups A1 and A2 (probably imported ceramics), they were made of several clay materials and fired at various 
temperatures. The production of these vases is not standardised, so they might either come from several workshops or from a single workshop following several protocols of production concerning clay materials and firing.

\section{Conclusion}

Thus, it seems from the chemical characterization that most of the ossuary vases of our study come from several Lugdunum workshops of cooking wares. They do not come from a specific workshop that specialised in the production of ossuary vases. The macroscopic observations made with the naked eye were supported by the apparent firing temperatures measured with the dilatometer: the ceramics which presented particularly damaged surfaces with characteristics of underfired ceramics were truly underfired as they were fired at temperatures below $700^{\circ} \mathrm{C}$. These underfired vases used as funerary pots are not found in later period funerary ensembles.

It is therefore possible to think that there was an opportunistic selection of vases for use in funerary contexts: sometimes taken directly from the kitchen dresser, sometimes bought expressly for funerary usage either in an undamaged state, or as producers' seconds.

It must be emphasised that this study was possible because in Lyon, both domestic and production contexts are very well known, allowing macroscopic and analytical comparisons with the finds of funerary sites. It is also clear that this is a preliminary case study that should be extended to other funerary sites in Lyon and then those of other regions.

\section{References}

Batigne Vallet, C. (dir.), (to be published), Céramiques de cuisine d'époque romaine en Rhône-Alpes et dans le sud de la Bourgogne ( $\mathrm{I}^{\mathrm{er}}$ av. J.-C.- $\mathrm{v}^{\mathrm{e}}$ s. apr. J.-C.) : morphologie, techniques et approvisionnement.

Batigne Vallet, C., 1999. Étude des céramiques culinaires et de leurs procédés de fabrication à Lyon à l'époque gallo-romaine : impératifs techniques et habitudes culturelles. Thèse de doctorat réalisée sous la direction de M. Picon, université Lyon 2.

Baxter, M., 1994. Exploratory Multivariate Analysis in Archaeology. Edinburgh University Press.

Baxter, M.J., 2003. Statistics in archaeology, London, Arnold, $292 \mathrm{p}$.

Blaizot, F. (dir.), 2009. Pratiques et espaces funéraires dans le centre et le sud-est de la Gaule durant l'Antiquité, Gallia, 66-1, CNRS éd., 383 p.

ArCHeOSCIENCES, revue d'archéométrie, 43(1), 2019, p. 97-106
Bonnet, Chr., Batigne Vallet, C., Brun, C., Barreto, F., 2016. Les vases ossuaires en céramique à Lyon de la fin du I ${ }^{\text {er }}$ siècle avant J.-C. au III ${ }^{\mathrm{e}}$ siècle après J.-C., SFECAG, Actes du congrès d'Autun, p. 175-202.

Collomb, Ph., Maggetti, M., 1996. Dissolution des phosphates présents dans des céramiques contaminées, Revue d'Archéométrie, 20: 69-75.

Cuomo di Caprio, N., Picon, M., 1999. La mesure des températures de cuisson des céramiques par dilatométrie, In M.A., RufRiba (ed.). 4t Congrés Europeu sobre Ceràmica Antiga, Actes del 5e Curs d'Arqueologia d'Andorra, nov. 1997. Govern d'Andorra, Andorra la Vella, p. 86-109.

Desbat, A., Genin, M., Lasfargues, J. (Ed.), 1996. Les productions des ateliers de potiers antiques de Lyon, Les ateliers précoces, CNRS, Gallia 53, 249 p.

Desbat, A., (Ed.), 1997. Les productions des ateliers de potiers antiques de Lyon, Les ateliers du ier s. après J.-C., CNRS Editions, Gallia 54: 1-117.

Desbat, A., 2010. L'artisanat antique à Lyon. In Pascale ChardronPicault (dir.), Aspects de l'artisanat en milieu urbain: Gaule et Occident romain, Revue archéologique de l'Est, suplément 28 : 55-74.

Maritan, L., Mazzoli, C., 2004. Phosphates in archaeological finds: implications for environmental conditions of burial. Archaeometry, 46: 673-683.

Maritan, L., Angelini, I., Artioli, G., Mazzoli, C., and Saracino, M., 2009. Secondary phosphates in the ceramic materials from Frattesina (Rovigo, North-Eastern Italy). Journal of Cultral Heritage, 10: 144-151.

Picon, M., 1984. Le traitement des données d'analyses. In: Datation-caractérisation des céramiques anciennes. Eds. T. Hackens and M. Schvoerer, PACT 10: 379-399.

Schneider, G., 2017. Mineralogical and chemical alteration, in A. Hunt (éd.), Oxford Handbook of Archaeological Ceramic Analysis: 162-180.

Thirion-Merle, V., 2014. Spectrométrie de fluorescence X, In Circulation et provenance des matériaux dans les sociétés anciennes, Dir. Dillmann P. et Bellot-Gurlet L., Collection Sciences Archéologiques, Paris, Editions des archives contemporaines, p. 291-297.

Tite, M.S., 1969. Determination of the firing temperature of ancient ceramics by measurement of thermal expansion: a reassessment, Archaeometry, 11: 131-143.

Waksman, S.Y., 2014. Etudes de provenance de céramiques, In Circulation et provenance des matériaux dans les sociétés anciennes, Dir. Dillmann P. et Bellot-Gurlet L., Collection Sciences Archéologiques, Paris, Editions des archives contemporaines, p. 195-215. 